THE ASTRophysical Journal SUPPLEMENT SERIES, 90:969-973, 1994 February

(c) 1994. The American Astronomical Society. All rights reserved. Printed in U.S.A.

\title{
TIME-DEPENDENT SIMULATION OF COSMIC-RAY SHOCKS, INCLUDING ALFVÉN TRANSPORT
}

\author{
T. W. JONES \\ Department of Astronomy, University of Minnesota, Minneapolis, MN 55455 \\ Received 1993 March 1; accepted 1993 May 13
}

\begin{abstract}
Time evolution of plane, cosmic-ray modified shocks has been simulated numerically for the case with parallel magnetic fields. Computations were done in a "three-fluid" dynamical model incorporating cosmic-ray and Alfvén-wave energy transport equations. Nonlinear feedback from the cosmic rays and Alfvén waves is included in the equation of motion for the underlying plasma, as is the finite propagation speed and energy dissipation of the Alfvén waves. Exploratory results confirm earlier, steady state analyses that found these Alfvén transport effects to be potentially important when the upstream Alfvén speed and gas sound speeds are comparable. As noted earlier, Alfvén transport effects tend to reduce the transfer of energy through a shock from gas to energetic particles. These studies show as well that the timescale for modification of the shock is altered in nonlinear ways. It is clear, however, that the consequences of Alfvén transport are strongly model dependent and that both advection of cosmic rays by the waves and dissipation of wave energy in the plasma will be important to model correctly when quantitative results are needed. Comparison is made between simulations based on a constant diffusion coefficient and more realistic diffusion models allowing the diffusion coefficient to vary in response to changes in Alfvén wave intensity. No really substantive differences were found between them.
\end{abstract}

Subject headings: cosmic rays - MHD — shock waves

\section{INTRODUCTION}

Diffusive transport of cosmic rays in shocks is mediated through resonant scattering by Alfvén waves. The dominant interactions probably involve Alfvén waves stimulated to propagate in the direction of cosmic rays streaming away from the shock itself. Although the existence of such waves is clearly implicit in all discussions of diffusive shock acceleration of cosmic rays, most treatments of this problem have neglected the motion of the Alfvén waves through the background plasma and the energy exchange between the Alfvén waves and the plasma; what we may term "Alfvén transport" effects. Several authors have pointed out, however, that Alfvén transport effects can place important controls on particle acceleration and shock dynamics when the Alfvén speed is significant compared to the upstream gas sound speed (e.g., Holman, Ionson, \& Scott 1979; Achterberg 1982; Völk, Drury, \& McKenzie 1984). To date, only steady shocks have been discussed in this context. As a first step to examine these issues in unsteady cosmic-ray shocks I have extended the time-dependent twofluid numerical methods for diffusive cosmic-ray transport employed in Jones \& Kang ( 1990) to include Alfvén transport in parallel MHD, sonic-mode shocks. More nearly complete Alfvén transport formalisms based on the diffusion-advection equation for the cosmic rays and general MHD are under development. In the present case the governing equations for the gas are

$$
\begin{aligned}
& \frac{d \rho}{d t}=-\rho \nabla \cdot \boldsymbol{u}, \\
& \frac{d \boldsymbol{u}}{d t}=-\frac{1}{\rho} \nabla\left(P_{g}+P_{c}+P_{w}\right),
\end{aligned}
$$

$$
\frac{d e}{d t}=-\frac{1}{\rho} \nabla \cdot\left\{\left(P_{g}+P_{c}+P_{w}\right) \boldsymbol{u}\right\}+\frac{1}{\rho}\left(P_{c}+P_{w}\right) \nabla \cdot \boldsymbol{u}+\frac{L}{\rho} .
$$

where $\boldsymbol{u}$ is the velocity of the gas, while $P_{g}, P_{c}=\left(\gamma_{c}-1\right) E_{c}$ and $P_{w}=E_{w} / 2$ are the pressures of the gas, the cosmic-rays and the waves, respectively; $d / d t$ is the Lagrangian time derivative, including the gas motion; and $e$ is the sum of gas thermal and kinetic energy per unit mass. The term $L$ represents nonadiabatic heating from dissipation of the Alfvén waves. Equations (1.1)-(1.3) differ from those in the usual two-fluid model for cosmic-ray transport only through the addition of the wave pressure, $P_{w}$.

The cosmic-ray energy density is followed through an energy conservation equation derived from the diffusion-advection equation given by Skilling (1975). It corrects the standard two-fluid expression (e.g., Drury \& Völk 1981) for the advected cosmic-ray energy flux and the work done by the cosmic rays on the gas for the fact that the cosmic rays are advected with the waves rather than the gas (McKenzie \& Völk 1982; Achterberg 1982);

$$
\frac{d E_{c}}{d t}=-\gamma_{c} E_{c}(\nabla \cdot \boldsymbol{u})+\nabla \cdot\left(\langle\kappa\rangle \nabla E_{c}-\boldsymbol{u}_{w} \gamma_{c} E_{c}\right)+\boldsymbol{u}_{w} \cdot \nabla P_{c},
$$

where $\langle\kappa\rangle$ is an energy weighted, mean diffusion coefficient and $u_{w}$ is the center-of-momentum velocity for the resonant Alfvén waves. Equation (1.4) is valid when this Alfvén centerof-momentum frame is independent of wavenumber, so that its motion is "fluid-like." If the wave propagation is unidirectional, then $u_{w} \approx v_{\mathrm{A}}$ in the direction of wave propagation. In a 
more general case, $u_{w}$ would lie between $\pm v_{\mathrm{A}}$. For the latter case one should properly include an energy gain term in equation (1.4) (and an analogous loss term in the energy equation for the Alfvén waves) to account for second-order Fermi acceleration; i.e., momentum diffusion. However, within the shock structures energy transfer due to momentum diffusion will generally be smaller than the spatial diffusion term by a factor less than $\sim\left(v_{\mathrm{A}} / u_{s}\right)^{2}$, where $u_{s}$ is the shock speed relative to the unshocked gas. So long as we limit ourselves to superalfvenic shocks, this should not have a major impact on energy transfer, although it might be significant in determining the form of the cosmic-ray momentum distribution.

Two-fluid methods have the limitations that they cannot follow the evolution of the cosmic-ray particle distribution self-consistently and that, consequently, one must a priori specify closure parameters such as the cosmic-ray adiabatic index, $\gamma_{c}$, and the mean diffusion coefficient, $\langle\kappa\rangle$, although they need not be constants. On the other hand, within the bounds implied by those caveats two-fluid models accurately describe the dynamics of the shock evolution and provide an efficient means to study the approach to dynamical equilibrium in such shocks. Our previous calculations comparing two-fluid and diffusion-advection calculations have shown two-fluid models to be an effective means to explore the general dynamical evolution of cosmic-ray mediated shocks (Kang \& Jones 1991; Kang, Jones, \& Ryu 1992; Jones \& Kang 1992).

To complete the formalism an energy equation for the Alfvén waves is needed. In the WKB approximation and under constraints similar to those applied to equation (1.4) the wave energy density obeys

$$
\frac{d E_{w}}{d t}=-\boldsymbol{u}_{w} \cdot \nabla P_{c}-\nabla \cdot\left(u_{w} E_{w}\right)-\frac{3}{2} E_{w} \nabla \cdot \boldsymbol{u}-L
$$

A full discussion of equations (1.1)-(1.5) with associated references is given in Jones (1993, hereafter J93). That paper also explores a range of plausible properties for Alfvén transport as applied to the evolution of plane shocks with magnetic fields parallel to the shock normal. The key Alfvén properties are (1) the model for the effective wave transport velocity, $\boldsymbol{u}_{w}$, as a function of space and time, (2) the model for wave energy dissipation, $L,(3)$ the ratio of the upstream value of the Alfvén speed, $v$, to the upstream gas sound speed, $c_{s o}\left(v_{A o}=c_{s o} / \sqrt{\beta}\right)$, and (4) the ratio of the upstream wave energy density to the magnetic energy density, $\alpha=E_{w o} / E_{B o}$.

Very briefly, the results discussed in J93 confirm the findings of earlier, steady state calculations showing the potential importance of Alfvén transport effects (e.g., Völk et al. 1984). Generally, Alfvén transport reduces the rate for transfer of energy from gas to cosmic rays and reduces the time-asymptotic postshock cosmic-ray pressure. For shocks of moderate strength (sonic Mach numbers up to a few tens), for values of $\beta \sim 1$, and for $u_{w} \approx v_{\mathrm{A}}$, the time-asymptotic, postshock cosmic-ray pressure can be reduced by more than an order of magnitude by these effects. An example illustrating this impact is shown in Figure 1. Details of those models are described in the next section and more completely in J93. Thus Alfvén transport has the potential to practically remove the strong modification of the shock structure by the cosmic rays. The time necessary for a modified shock to approach dynamical equilibrium is also changed, but in nonlinear ways that sometimes increase and sometimes decrease the "equilibrium" time compared to that found in similar calculations with negligible Alfvén transport. It was clear from the time-dependent simulations that influences both from the advection by waves and from wave energy dissipation are important contributors to the dynamical evolution of cosmic-ray modified shocks. The net influence of Alfvén transport depends sensitively upon the details of the generation, dissipation, and especially advection of Alfvén waves in the vicinity of shocks. In addition, it was clear that Alfvén transport properties on both sides of the shock are important whenever one considers unsteady shocks. For example, in the event that streaming by cosmic-rays downstream of the shock transition stimulates Alfvén waves propagating downstream as well, the net effects of Alfvén transport can be greatly increased relative to models in which the waves always face upstream.

\section{EVOLUTION OF THE MEAN DIFFUSION COEFFICIENT}

One effect that was not discussed in detail in J93 was the influence of the spatial and temporal evolution of the diffusion coefficient, $\langle\kappa\rangle$ due to changes in the Alfvén wave intensity according to equation (1.5). In theory the diffusion coefficient depends upon the Alfvén wave energy density, according to

$$
\langle\kappa\rangle \propto \frac{E_{B}}{E_{w}},
$$

where $E_{B}=B^{2} /(8 \pi)$, is the total magnetic field energy density. Since the calculations described in $\mathrm{J} 93$ showed increases in $E_{w}$ by factors $\sim 10$ across strong shocks, whereas $E_{B}$ remains constant in parallel shocks, equation (2.1) would imply large decreases in $\langle\kappa\rangle$ as material passes through. In the simulations discussed in $\mathrm{J} 93\langle\kappa\rangle$ was taken to be a constant in order to isolate other important physical effects. The question would be what impact various properties of $\langle\kappa\rangle$ have on the dynamical evolution of the shocks under study. On the one hand, $\langle\kappa\rangle$ determines the characteristic length scale for the shock precursor, $x_{d} \sim\langle\kappa\rangle / u_{s}$, and the timescale, $t_{d}=x_{d} / u_{s}$, for dynamical evolution of a shock; i.e., the time necessary to approach an approximate dynamical equilibrium. Those scales will be reduced as $\langle\kappa\rangle$ becomes smaller, as will the time for individual cosmic-ray particles to be accelerated. On the other hand, simple arguments suggest that the value of $\langle\kappa\rangle$ cannot influence steady state solutions to equations $(1.1)-(1.5)$. Of course, on sufficiently long timescales the details of the diffusion coefficient, and especially its momentum dependence must influence such issues as the eventual escape of very high energy cosmic-ray particles. For that reason and others, as well, steady two-fluid models are probably not very realistic as detailed models. Strongly modified cosmic-ray shocks are perhaps never in true equilibrium, anyway, since they seem to be subject to various instabilities (e.g., Ryu, Kang, \& Jones 1993). Thus the specific question more relevant to the present explorations is what influence the properties of $\langle\kappa\rangle$ in equation (2.2) may have on the timescales and on the properties of cosmicray modified shocks as they approach dynamical equilibrium.

To allow simple examination of these questions, I present 

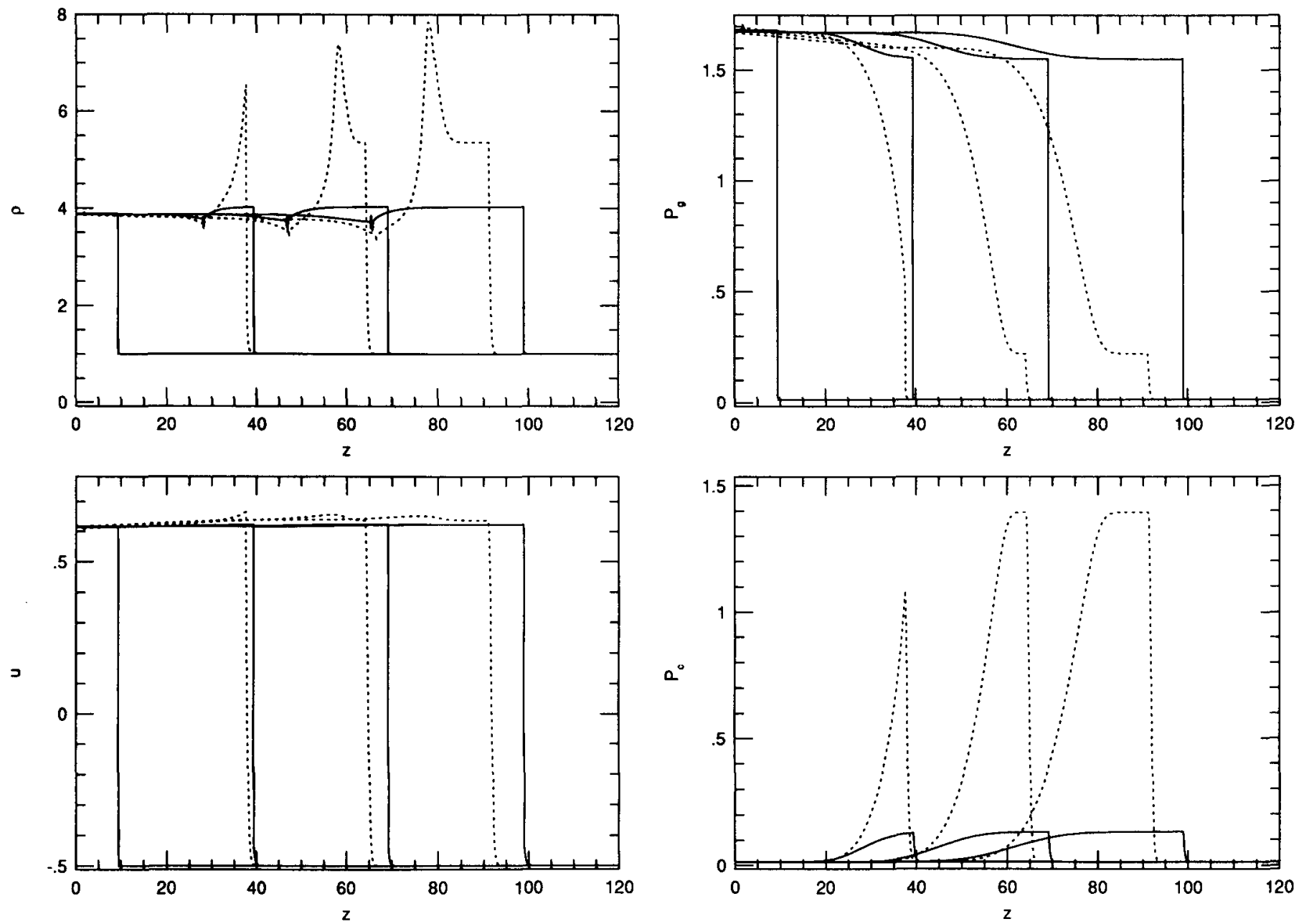

FIG. 1.-Evolution of two plane shocks differing only in the presence or absence of Alfvén transport effects. The solid curves represent model A, described in the text and also shown in Fig. 2. The dotted curves represent the behavior for the same model with $\beta=10^{4}$, so that the Alfvén transport terms in eqs. (1.1)-(1.4) are negligible. Density, gas velocity, gas pressure, and cosmic-ray pressure are shown at times $t=0,30,60$, and 90 . The nominal diffusion times and lengths for these models are $t_{d}=0.156$ and $x_{d}=0.233$. Note, in addition to the large change in $P_{c}$ between the two models the substantial change in compression and shock speed.

results from three shock simulations (labeled below as models $\mathrm{A}, \mathrm{B}$, and C) that differ only in the assumed character for the mean diffusion coefficient, $\langle\kappa\rangle$. The other, common properties are as follows: all the models assume a uniform initial (upstream and downstream) cosmic-ray pressure, $P_{c o}=P_{g o}=$ $1.35 \times 10^{-2}$, a gas Mach number for the initial shock $M=10$, a ratio of upstream sound speed to Alfvén speed, $\sqrt{\beta}=1$, and an upstream ratio of energy density in Alfvén waves to total magnetic field, $\alpha=0.5$. The cosmic-ray adiabatic index is assumed to be $\gamma_{c}=1.4$, which is approximately what one would infer for galactic cosmic rays if the observed power-law momentum distribution extends to nonrelativistic energies. That choice is not critical to our results, however. I assumed in each simulation that $\left|\boldsymbol{u}_{w}\right|=v_{\mathrm{A}}$, with the sign determined by the local cosmicray streaming direction; i.e., so that $\boldsymbol{u}_{w} \cdot \nabla P_{c}<0$. I also assumed a "local equilibrium" model for the wave dissipation rate,

$$
L=-\boldsymbol{u}_{w} \cdot \nabla P_{c}
$$

All the simulations were initiated with a preexisting gas shock discontinuity propagating to the right at unit speed in the frame of the grid. That corresponds in the frame of the upstream gas to $u_{s}=1.5$. Numerical methods were those described in detail in $\mathrm{J} 93$.

For model $A$ the diffusion coefficient was taken to be the constant $\langle\kappa\rangle=0.35$. This model is thus identical to the model 3 in J93, except for the value of $\alpha$. For model B I took $\langle\kappa\rangle=$ $1.4 / \rho$, while for model $\mathrm{C}\langle\kappa\rangle=1.4 E_{w o} / E_{w}$. The behavior for $\langle\kappa\rangle$ in model $B$ follows that used by a number of authors before (e.g., Drury \& Falle 1986; Jones \& Kang 1990). It was originally introduced to avoid the possible development of an acoustic instability in strongly cosmic-ray modified shocks, but also provides a crude way to allow for the effects indicated by equation (2.1). Model $\mathrm{C}$ is, of course, directly based on equation (2.1).

The evolution of all three shocks A-C is illustrated in Figure 2 . It is readily apparent that the differences among them are, in fact, minor. Because $\langle\kappa\rangle$ becomes smaller in models B and C, 

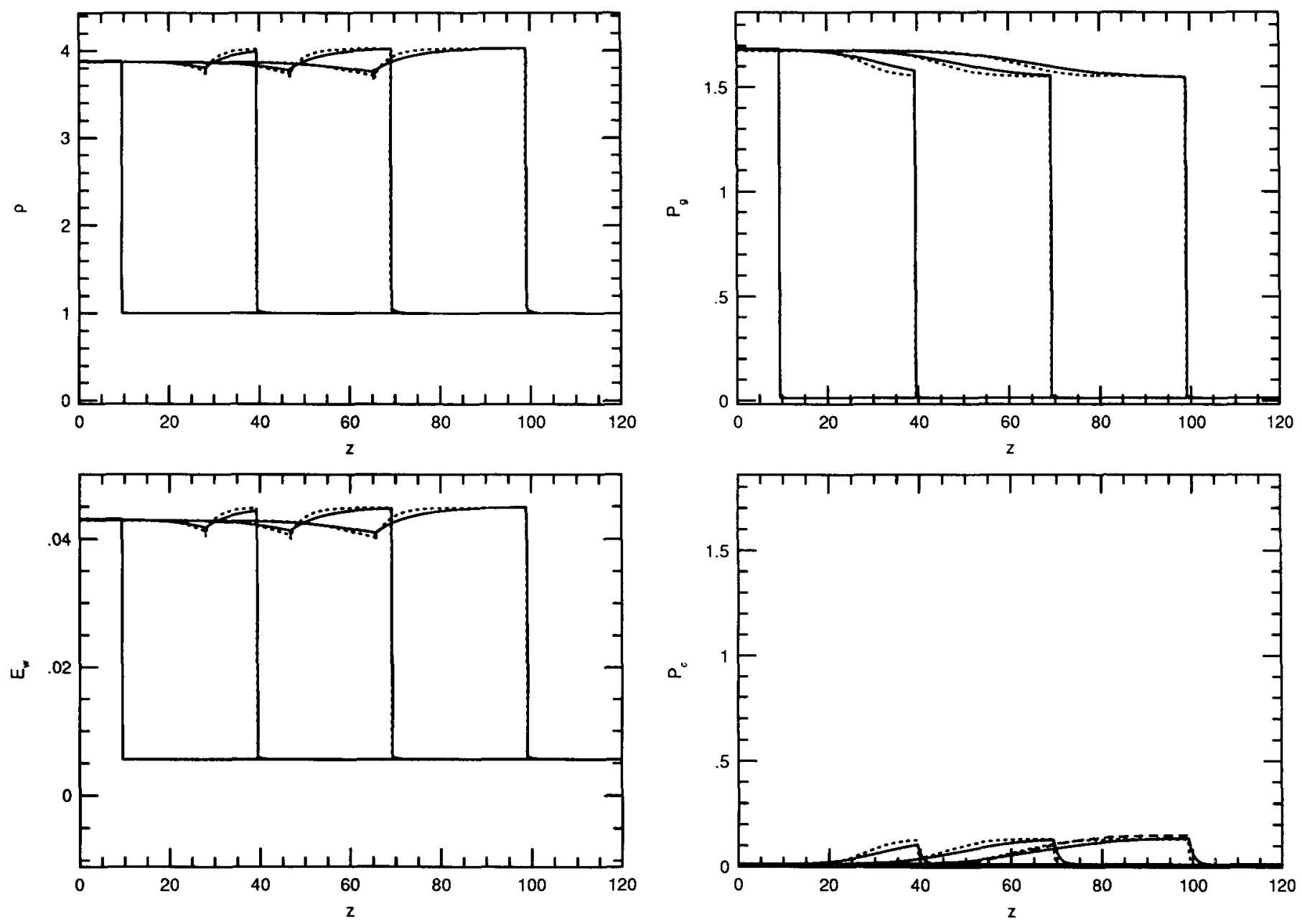

FIG. 2.-Evolution of three shocks using different models for the diffusion coefficient, $\langle\kappa\rangle$. Each simulation assumes $\gamma_{\mathrm{c}}=1.4, \beta=1, \alpha=1 / 2, M=10$, $P_{c o}=P_{g o}$. The solid curve represents the case $\langle\kappa\rangle=0.35$ (model A). The dotted curves correspond to $\langle\kappa\rangle=1.4\left(E_{w o} / E_{w}\right)$ (model C). The dashed curve $\left(P_{c}\right.$ only) shows the case $\langle\kappa\rangle=1.4 / \rho(\operatorname{model} B)$. The asymptotic shock properties are the same to within the limits of numerical accuracy at finite time and resolution. Differences in $P_{c}$ within the precursors are due to differences in the effective diffusion length, $x_{d}$.

the effective length and timescales, $x_{d}$ and $t_{d}$ are reduced. Thus the width of the shock precursor is smaller, and the shocks reach dynamical equilibrium more quickly. The former effect is best seen in the plot of $P_{c}$, while the latter is evident in all the variables, since it shows equilibrium postshock properties farther behind the "current" shock positions. For all times shown, however, all the shocks have actually closely approached equilibrium in their immediate postshock properties.

On the other hand, and perhaps most important to the issues addressed in the study by $\mathrm{J} 93$, all three models produce very similar time asymptotic values for $P_{g}$ and $P_{c}$; i.e., the "efficiency" of particle acceleration and the degree of shock modification by cosmic rays is the same to within the limits of the numerical scheme ( see $\mathbf{J} 93$, for a discussion of those limits). Thus, for qualitative studies of this type, involving plane shocks and two-fluid models, the detailed behavior of $\langle\kappa\rangle$ does not seem to be critical. However, for unsteady, nonplanar shocks, such as supernova remnants (e.g., Jones \& Kang 1992; Jones \& Kang 1993) the specific properties of $\langle\kappa\rangle$ are more significant.

\section{CONCLUSIONS}

Alfvén transport effects in "low $\beta$ " plasmas can be important to the transfer of energy from the thermal plasma to highenergy cosmic-ray particles. Because streaming by the cosmic rays tends to follow the propagation of Alfvén waves resonantly interacting with them, and because the speed of that propagation in parallel shocks tends to change across the shock transition, both the energy gain by a particle and the residence time by particles near the shock are modified from calculations which assume that cosmic rays are advected exactly with the gas. Furthermore, dissipation of wave energy in the gas transfers energy from cosmic-rays to the gas and preheats gas entering a shock, tending for both reasons to reduce the net efficiency of energy transfer to cosmic-rays in the shocks. In an associated paper (J93). I discussed in detail these effects in the context of the dynamical evolution of plane shock structures, emphasizing the sensitivity to uncertain details about models for Alfvén transport. Those computations are based on a "three-fluid" model for the dynamical interactions of the gas, the cosmic-rays, and the Alfvén waves in shocks with magnetic 
fields parallel to the shock normal. That discussion did not consider consequences of using a self-consistent model for the diffusion coefficient which allowed for its dependence upon the local intensity of Alfvén waves. Rather, in an effort to isolate other, clearly important model features for Alfvén transport, J93 presented only calculations using constant mean diffusion coefficients. In the present paper I have presented a comparison of one such model with models that allow for changes in the diffusion coefficient in response to changes in the Alfvén wave energy density as the waves are advected through a shock. These computations are still done within a three-fluid model, but in one case do include nonlinear modifications to the waves due to the cosmic-rays and to wave dissipation within the plasma. Although there are expected changes in the characteristic diffusion lengths and times associated with the shock structure, the more complicated behavior of the diffusion coefficient does not appear to alter in any discernible way the time-asymptotic properties of the postshock flow. Most important in this regard, it does not seem to alter the expected net efficiency of energy transfer to cosmic-rays within a transition.

Of course, treatment of diffusive shock acceleration with Alfvén transport based on a more complete formalism using the momentum dependent, diffusion-advection equation will be necessary before one could possibly extend that conclusion to any discussion of the properties of the cosmic-ray particle distribution. To that end I have under development an effort to simulate the evolution of cosmic-ray-mediated shocks using the diffusion-advection equation and also including nonlinear magnetohydrodynamical (MHD) gas dynamics (see Frank, Jones, \& Ryu 1994). The much greater complexity of those treatments, however, will certainly cause us to look back for benchmarks to simpler models, such as those discussed in $\mathbf{J} 93$ and the present paper.

This work was supported in part by NASA through grant NAGW-2548, the NSF through grant AST-9100486 and by the University of Minnesota Supercomputer Institute.
Achterberg, A. 1982, A\&A, 98, 195

Drury, L. O'C., \& Falle, S. A. E. G. 1986, MNRAS, 223, 353

Drury, L. O'C., \& Völk, H. J. 1981, ApJ, 248, 344

Frank, A., Jones, T. W., \& Ryu, D. 1994, ApJS, 90, 969

Holman, G., Ionson, J., \& Scott, J. 1979, ApJ, 228, 576

Jones, T. W. 1993, ApJ, 413, 619 (J93)

Jones, T. W. \& Kang, H. 1990, ApJ, 363, 499

1992, ApJ, 396, 586

\section{REFERENCES}

Jones, T. W. 1993, ApJ, 402, 560

Kang, H., \& Jones, T. W. 1991, MNRAS, 249, 439

Kang, H., Jones, T. W., \& Ryu, D. 1992, ApJ, 385, 193

McKenzie, J. F., \& Völk, H. J. 1982, A\&A, 116, 191

Ryu, D., \& Jones, T. W. 1994, in preparation

Ryu, D., Kang, H., \& Jones, T. W. 1993, ApJ, 405, 199

Skilling, J. 1975, MNRAS, 172, 557

Völk, H., Drury, L., \& McKenzie, J. 1984, A \& A, 130, 19 